\title{
Pathological and Clinical Characteristics of Triple Negative Breast Cancer and Disease Relapse
}

\author{
Duaa Knaj $^{1,}$, Michael Georgeos ${ }^{2}$ \\ ${ }^{1}$ Department of Oncology, Faculty of Medicine, Tishreen University, Lattakia, Syria \\ ${ }^{2}$ Oncology Department, Oncology Center, Tishreen University Hospital, Lattakia, Syria
}

Email address:

Duaa.Knaj@tishreen.edu.sy (D. Knaj)

${ }^{*}$ Corresponding author

\section{To cite this article:}

Duaa Knaj, Michael Georgeos. Pathological and Clinical Characteristics of Triple Negative Breast Cancer and Disease Relapse. International Journal of Clinical Oncology and Cancer Research. Vol. 5, No. 4, 2020, pp. 78-81. doi: 10.11648/j.ijcocr.20200504.11

Received: August 29, 2020; Accepted: September 14, 2020; Published: November 19, 2020

\begin{abstract}
Background: Breast cancer is the most common cancer and an important cause of death in women. It has psychological and social repercussions in patients. Objective: We aimed to assess triple negative breast cancer characteristics, patterns and time of disease relapse. Patients and methods: We reviewed the clinical files and pathology reports of 100 patients who had primary triple-negative breast cancer between 2013-2017 at the oncology center, Tishreen University Hospital in Lattakia, Syria. We collected the information about (age at diagnosis, histological subtype of tumor, histological grade, tumor size, tumor stage, lymph node status), which was taken at the time of diagnosis. We used SPSS in this research, and Chi-square test was used to compare variables. Results: We found that most of patients $(85 \%)$ were 40 years of age and older when diagnosed with breast cancer. The most common histological subtype was invasive ductal carcinoma IDC (84\%). Regarding the size of the primary tumor, the majority (72\%) were T2. According to lymph node status at diagnosis, 41 patients $(41 \%)$ were $\mathrm{N} 0,(33 \%)$ of total were $\mathrm{N} 1$. We found grade 2 in $52 \%$, followed by grade3 $(41 \%)$. For the tumor clinical stage, it was according to AJCC $7^{\text {th }}$ edition, stage II (63\%) and stage III in (30\%). We found that 32 patients had a recurrence (32\%) while $(68 \%)$ had no recurrence during the follow-up period. As for the development of recurrence, $(40.6 \%)$ had visceral recurrence; the most pattern was hepatic (61.5\%) then pulmonary (38.5\%). Recurrence occurred in (22 of 32) patients during the first 2 years $(68.7 \%)$. We found a significant association between lymph node status and recurrence in patients with TNBC $(\mathrm{P}=0.004)$ Conclusion: $\mathrm{T}$ and $\mathrm{N}$ were important predictive factors for relapse in patients with triple-negative breast cancer, which tends to recur often during the first two years of diagnosis with distant metastases as well as localized recurrence.
\end{abstract}

Keywords: Triple Negative Breast Cancer, Characteristics, Relapse

\section{Introduction}

Breast cancer has psychological and social repercussions in patients. It is the most common cancer and the second cause of death in women after lung cancer [1]. It is considered a heterogeneous disease that includes a group of subtypes. Triple-negative breast cancer is a special subtype that constitutes about (10-20) \% of all breast cancers [2]. Histologically characterized by the lack of expression of estrogen receptor (ER), progesterone receptor (PR), and human epidermal growth factor receptor 2 (HER2) [3]. TNBC has several clinical and pathological characteristics that are considered prognostic factors, which contribute to identify patients' risk groups and make appropriate treatment decisions. TNBC tends to have a higher risk of recurrence and aggressiveness than other subtypes, most of relapses usually occur within the first 5 years of diagnosis [4]. The high risk of distant recurrence in the visceral organs is an important issue in these tumors, and this makes periodic monitoring something necessary for early detection of relapse and for appropriate treatment with the aim of improving patient's long-term survival.

\section{Patients and Methods}

This study included 100 patients with primary triplenegative breast cancer between 2013-2017 at the Oncology 
Center, Tishreen University Hospital in Lattakia-Syria. The diagnosis of triple-negative breast cancer was confirmed histologically by immunochemical staining. The study was performed in a retrospective manner; we reviewed the medical files and histopathology reports for each patient then a questionnaire was drawn up with the following clinicpathology information, which was taken at the time of diagnosis: age at diagnosis, tumor histological subtype, primary size of the tumor (in centimeters), histological grade, stage of the tumor, lymph node status. The patients were followed up since the diagnosis and for at least a period of 4 years on average. During the follow-up, the incidence of recurrence was recorded, including which was seen at diagnosis. We divided the patients into two groups according to the time of recurrence: during the first 2 year, and recurrence after 2 years. Depending on the pattern of recurrence, it was classified into visceral (pulmonary, hepatic), loco-regional, bone and brain recurrence. Data were collected and documented.

\section{Statistical Analysis}

We performed the analysis using the Statistical Package for Social Sciences (SPSS). P was considered statistically significant if less than $0.05(\mathrm{p}<0.05)$. The Chi-square test was used to compare variables.

\section{Results}

\subsection{Characteristics of TNBC}

Data were collected from 100 patients with triple-negative breast cancer. Eighty-five patients $(85 \%)$ were 40 years- old and older when diagnosed with breast cancer, $15 \%$ were less than 40 years old with a median age of 53 years (range 2879). The most common histological subtype was invasive ductal carcinoma IDC in $84 \%$ of the cases, and invasive lobular carcinoma ILC in $10 \%$, and the rest of patterns were distributed between medullary and intra-ductal carcinoma in $6 \%$ of the cases. The World Health Organization classification of breast tumors was adopted (Figure 1).

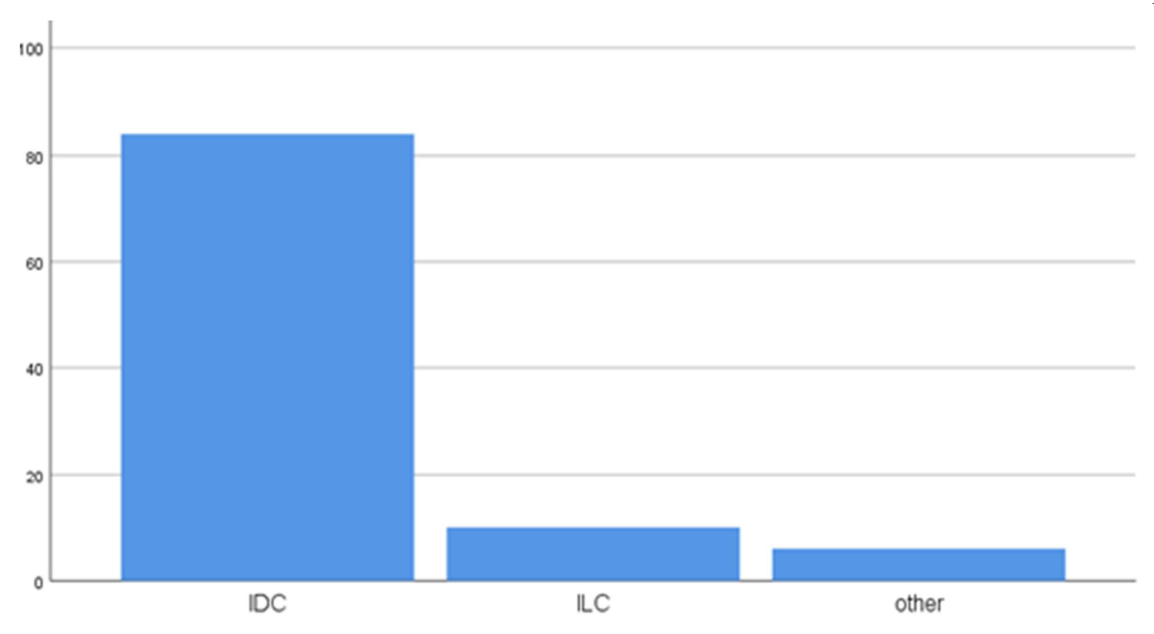

Figure 1. Histological subtypes of TNBC.

Regarding the size of the primary tumor, the majority (72\%) were $\mathrm{T} 2,12 \%$ were $\mathrm{T} 3, \mathrm{~T} 1$ in $10 \%$ of patients, with regard to $\mathrm{T} 4$ it was only in $6 \%$. According to lymph node status at diagnosis, 41 patients $(41 \%)$ were N0, $(33 \%)$ of total were N1, N2 was found in 19 patients (19\%), and only 7 cases were classified as N3 (7\%), Figure 2.

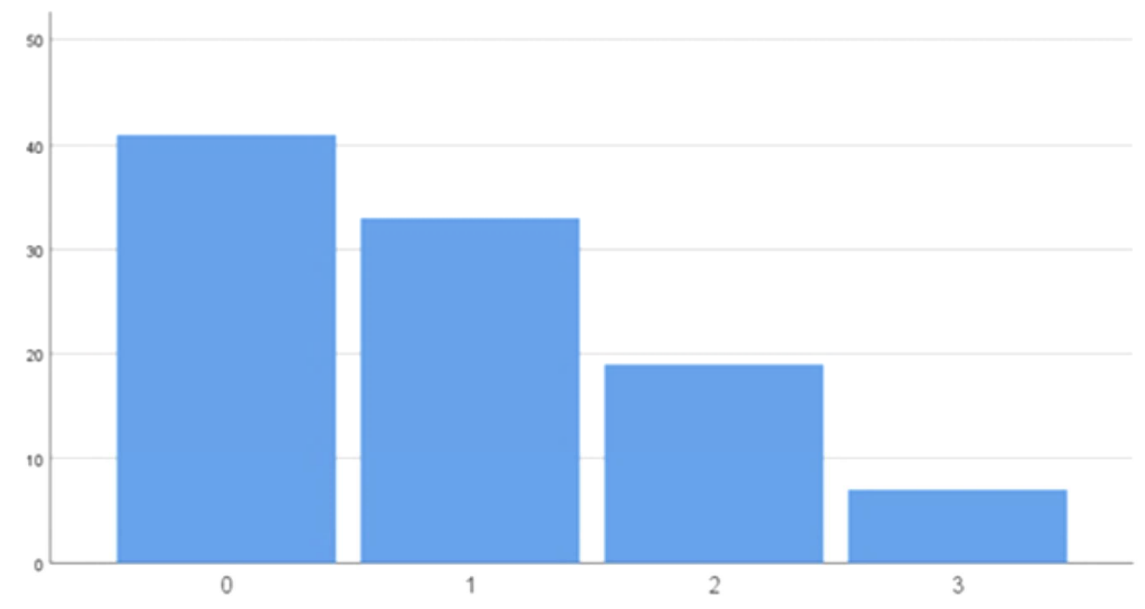

Figure 2. Lymph node status of (100) TNBCS. 
It was adopted Nottingham Histological Score to determine the histological grade of the tumor [5], we found grade 2 in $52 \%$, followed by grade $3(41 \%$ ), while only $7 \%$ were grade 1 . For the tumor clinical stage, it was according to AJCC $7^{\text {th }}$ edition (American Joint Committee on Cancer) [6], stage II (63\%), stage III in 30\%, stage I (6\%), and stage IV was only in $1 \%$.

\subsection{Recurrence}

Through the follow-up of the patients and based on the recorded data, the results showed that 32 patients of 100 had a recurrence $(32 \%)$, while $(68 \%)$ did not have a recurrence during the follow-up period. As for the development of recurrence, $(40.6 \%)$ had visceral recurrence; the most pattern was hepatic $(61.5 \%)$ then pulmonary (38.5\%), hepatic -pulmonary recurrence occurred in $23 \%$ of patients. As for local recurrence, it occurred in 34.3\% of patients. Skeletal recurrence was observed in $15.6 \%$. Distant metastases in the central nervous system were located in $9.3 \%$ of patients.

In order to know the time of recurrence in triplenegative breast cancer and according to the previous classification into two groups: (22 of 32) patients were diagnosed during the first 2 years $(68.7 \%)$, in 10 patients relapse occurred after the first two years (31.2\%). Based on Chi-square test, a correlation was found between the number of involved lymph nodes and the incidence of recurrence $(\mathrm{P}=0.004)$, as it was higher with increased lymph nodes involvement.

\section{Discussion}

Our study included 100 patients of TNBC diagnosed and treated in Tishreen University Hospital table 1. We found that 32 patients had a recurrence (32\%) while 68\% had no recurrence during the follow-up period. Most of the patients $(85 \%)$ were 40 years of age an older when diagnosed with breast cancer, and (15\%) of them were less than 40 years-old, the median age was 53 years.

Distant metastases in addition to local recurrence after treatment are the most common cause of death in breast cancer [7]. In our study, distant relapse occurred in (65\%) of patients and localized relapse in approximately (34\%). We found that $(40.6 \%)$ of relapsing patients had relapses in the visceral organs (13 of 32). The most common places were $(61 \%)$ hepatic recurrence, $(38 \%)$ pulmonary recurrence, and in 3 patients there was a combined hepatic-pulmonary recurrence $(23 \%)$, the bones were the subject of relapse in 5 patients $(15.6 \%)$ and the brain $(9.3 \%)$ in 3 patients table 2 . That is, the visceral organs were the common site of recurrence among the largest proportion of patients. This is consistent with a study conducted between 1987 and 1997 in Toronto on 1600 patients with breast cancer [8], where TNBC patients were more prone to distant relapse $(33.9 \%)$ with visceral pattern in most prevalent, followed by local relapse in $14 \%$ of patients.

Table 1. Characteristics of triple negative breast cancer.

\begin{tabular}{|c|c|}
\hline & $\mathrm{N}=100, \mathrm{n}(\%)$ \\
\hline \multicolumn{2}{|c|}{ Age at diagnosis } \\
\hline$<40$ & 15 \\
\hline $40-50$ & 26 \\
\hline $51-55$ & 21 \\
\hline$>55$ & 38 \\
\hline \multicolumn{2}{|c|}{ Tumor histology subtype } \\
\hline IDC & 48 \\
\hline ILC & 10 \\
\hline Other & 6 \\
\hline \multicolumn{2}{|c|}{ Histology grade } \\
\hline 1 & 7 \\
\hline 2 & 52 \\
\hline 3 & 41 \\
\hline \multicolumn{2}{|c|}{ Tumor size $(\mathrm{cm})$} \\
\hline $\mathrm{T} 1$ & 10 \\
\hline $\mathrm{T} 2$ & 72 \\
\hline $\mathrm{T} 3$ & 12 \\
\hline $\mathrm{T} 4$ & 6 \\
\hline \multicolumn{2}{|c|}{ Lymph nodes status } \\
\hline No & 41 \\
\hline N1 & 33 \\
\hline N2 & 19 \\
\hline N3 & 7 \\
\hline \multicolumn{2}{|c|}{ Tumor stage } \\
\hline I & 6 \\
\hline II & 63 \\
\hline III & 30 \\
\hline IV & 1 \\
\hline \multicolumn{2}{|c|}{ Distant metastasis } \\
\hline M0 & 68 \\
\hline M1 & 32 \\
\hline
\end{tabular}

Recurrence was studied in TNBC with 841 patients in Italy between $1994-2015$ [9], where metastases were found at diagnosed in $(1.2 \%)$ of patients mostly hepatic $(80 \%)$ and this is consistent with our results. According to the previous analysis [8], the time of relapse in TNBC is usually during the first 1-3 years of diagnosis, and the risk of relapse may continue to occur even after 5 years. In our study, the most recurrences occurred within the first two years of diagnosis (22 of 32 ) with a rate of $68.7 \%$, and in 10 patients, the recurrence occurred after two years $(31.2 \%)$.

Table 2. Patterns of recurrence of TNBC.

\begin{tabular}{llll}
\hline & & Number & Percentage (\%) \\
\hline \multirow{3}{*}{ Visceral } & Liver & 8 & 61.54 (of 13) \\
& Pulmonary & 5 & 38.46 (of 13) \\
& Both & 3 & 23.08 (of 13) \\
Bone & Total & 13 & 40.63 \\
Brain & & 5 & 15.63 \\
Loco regional & & 3 & 9.37 \\
Total & 11 & 34.37 \\
\hline
\end{tabular}


This also agrees with a study of TNBC patients between $2005-2015$, which included 197 patients that relapse occurred in $16 \%$ of patients at early time, it was in $66 \%$ of patients within two years of diagnosis. During the first 3 years, $75 \%$ of relapses occurred [10]. This supports the fact that most recurrences in TNBC are early and within 5 years of diagnosis [11]

The histological grade of tumor is considered an important prognostic factor in breast cancer [12]. In our study, the histological grade was generally high [13]. Grade 2 was in 52\% of patients and grade 3 was found in $41 \%$. We did not find a statistical correlation between grade and recurrence of TNBC patients in our study $(\mathrm{P}=0.235)$. This correlation was also not found in previous studies $[9,10]$.

We found the most tumors with a size of $2-5 \mathrm{~cm}$ (T2) in $72 \%$ of patients, followed by T3 in $12 \%$ [14]. We observed that the incidence of relapse increased with the size of tumor increased $(p=0.106)$. There was no statistical relationship between size and recurrence. This result was found in two analyses $[8,9]$ that T2 was the majority and an increase in tumor size $>2 \mathrm{~cm}$ was associated with an increased risk of recurrence with a value of $p$ $<0.001$ [10].

Regarding the status of lymph nodes at diagnosis, most of the tumors were negative lymph nodes N0 (41\%), followed by $\mathrm{N} 1(33 \%)$ of patients [15]. We found a significant association between lymph node status and recurrence in patients with TNBC $(\mathrm{P}=0.004)$. This corresponds to the result of the analysis [10] in which the pathological node stage was significantly prognostic $(\mathrm{P}<0.001)$ as in our study. The majority in the Italian study were N0 (52\%) then N1 (22\%) [9].

According to AJCC 7th edition, the most common clinical stage was stage II $(63 \%)$ followed by stage III $(30 \%)$ of patients. In our study, we found an association between TNM stage and cancer recurrence in TNBC patient. This was found in the previous analysis $[9,10]$. Grade 2 was predominant in patients with TNBC in most previous studies.

\section{Conclusion}

$\mathrm{T}$ and $\mathrm{N}$ were important predictive factors for relapse in patients with TNBC. Through our study, we found that triplenegative breast cancer tends to recur often during the first two years of diagnosis, with distant metastases as well as localized recurrence. This aggressive behavior of cancer presupposes the trend towards further activation of the role of PET imaging, which may detect relapse not detected by other imaging methods earlier. In addition, to search for clinical and pathological factors predictive of relapse in an attempt to develop early treatment and decrease the negative effects of this cancer in terms of morbidity and mortality.

\section{References}

[1] Canadian Cancer Society's Advisory Committee on Cance Statistics. Canadian Cancer Statistics 2017. Toronto, ON: Canada Cancer Society; 2017.

[2] Schmadeka R, Harmon BE, Singh M. Triple-negative breast carcinoma: current and emerging concepts. Am J Clin Pathol 2014; 141: 462-77.

[3] Pierobon M, Frankenfeld CL. Obesity as a risk factor for triple- negative breast cancers: a systematic review and metaanalysis. Breast Cancer Res Treat 2013; 137: 307-14.

[4] Dent R, Trudeau M, Pritchard KI, Hanna WM, Kahn HK, Sawka CA, Lickley LA, Rawlinson E, Sun P, Narod SA. Triple-negative breast cancer: clinical features and patterns of recurrence. Clin Cancer Res. 2007; 13: 4429-34.

[5] Elston CW, Ellis JO: Pathological prognostic factors in breast cancer. 1. The value of histological grade $1 \mathrm{n}$ breast cancer: Experience from a long study with long-term follow -up. Histopathology 1991, 19: 40410.

[6] Amin MB, Edge FL, Edge SB, et al. The eighth edition AJCC Cancer Staging Manual: continuing to build a bridge from a population-based to a more "personalized" approach to cancer staging. CA Cancer J Clin. 2017; 67: 93-99.

[7] T. Tarver, Cancer facts \& figures 2012. American cancer society (ACS), J. Consum. Health Internet 16 (2012) 366e367.

[8] Clin Cancer Res 2007; 442913 (15) August 1, 2007.

[9] Urru et al. BMC Cancer (2018) 18: 56. DOI 10.1186/s12885017-3969-y.

[10] $10.21203 /$ rs.2.16295/v1.

[11] Lin NU, Vanderplas A, Hughes ME, et al. Clinicopathologic features, patterns of recurrence, and survival among women with triple-negative breast cancer in the National Comprehensive Cancer Network. Cancer 2012; 118: 5463-72.

[12] Rakha EA, Reis-Filho JS, Baehner F, Dabbs DJ, Decker T, Eusebi V, Fox SB, Ichihara S, Jacquemier J, Lakhani SR, et al. Breast cancer prognostic classification in the molecular era: the role of histological grade. Breast Cancer Res. 2010; 12: 207.

[13] Foulkes WD, Smith IE, Reis-Filho JS. Triple-negative breast cancer. N Engl J Med. 2010; 363: 1938-48.

[14] National Comprehensive Cancer Network. Breast Cancer (Version 2.2019). https://www.nccn.org/professionals/physician_gls/pdf/breast.p df. Accessed August 1, 2019.

[15] Brierley JD, Gospodarowicz MK and Wittekind C: TNM Classification of Malignant Tumours. 8th. Oxford; UK: Wiley Blackwell: 2017. 